\title{
INTEGRATED MANAGEMENT OF SUNFLOWER NECROSIS DISEASE
}

\author{
Shirshikar, S.P.* \\ Oilseeds Research Station, Latur-413 512 (M.S.) India
}

Received: June 26, 2007

Accepted: August 15, 2008

\begin{abstract}
SUMMARY
Sunflower necrosis disease (SND) is a new threat for sunflower cultivation in India. The disease was observed during 1997 in Karnataka, a major sunflower growing state of India. Later, its occurrence was reported from almost all sunflower growing states of India, posing threat to sunflower cultivation.

Presently no reliable resistant sources are available. The disease being viral in nature is very much difficult to combat by single approach. At Oilseeds Research Station, Latur (M.S.), India, three-year (2002-2004) field experiments revealed that if the sunflower crop is bordered with sorghum, and sunflower seeds treated with imidacloprid (Gaucho 70 W.S., $5 \mathrm{~g} / \mathrm{kg}$ ) along with sunflower spraying with imidacloprid (Confidor 200 S.L., 0.05 percent, three sprays at 15,30 and 45 days after sowing), the incidence of sunflower necrosis disease can be minimized.
\end{abstract}

Key words: sunflower necrosis disease, border crop, imidacloprid, seed treatment, disease management

\section{INTRODUCTION}

Sunflower (Helianthus annuus L.) is a major oil seed crop in India. More than 30 diseases have been reported on sunflowers worldwide (Gulya et al., 1994). However, under Indian conditions, only few of them are of common occurrence. These include Alternaria blight, rust and downy mildew. Among these, the downy mildew problem has been solved to a great extent by undertaking surveys (Shirshikar, 1997), releasing mildew-resistant hybrids through extensive screening at Oilseeds Research Station, Latur (Shirshikar, 2005a) and advocating chemical seed treatment (Shirshikar, 2005b).

However, recently a new destructive disease named sunflower necrosis disease (SND) has been noticed on sunflowers. The disease was observed for the first time

* Corresponding author: Phone (office) (+ 91-02382) 245294, (home) (+ 91-02382) 226615 
in 1997 in seed production area of Bagepally village in Kolar District of Karnataka State of India (Singh et al., 1977). In subsequent years, outbreaks of this disease in major sunflower-growing states of India, especially Andhra, Karnataka and Maharashtra, have virtually threatened the sunflower cultivation and yield losses ranging from 30 to 100 percent have been reported (Chander Rao et al., 2000).

The disease is of recent origin and reliable resistance sources have not been found. The disease is caused by tobacco streak virus (Prasada Rao et al., 2000, Bhat et al., 2002) and it was found to be transmitted by thrips (Harvir Singh, 2005). It is difficult to combat the disease through single approach as the disease is of viral origin. Most of the sunflower hybrids currently under cultivation in India have shown various degrees of susceptibility to the disease.

Recently, adjusting sowing period has been proposed for management of the disease. Also, sunflower crop sowing in post rainy season (September onwards) was found to be beneficial for minimizing necrosis incidence (Shirshikar, 2003).

A three-year field trial was conducted during kharif season at Oilseeds Research Station, Latur (M.S.), India, to work out integrated disease management strategy for this disease. The main objective of the experiment was to determine the effect of border crop, combinations of seed treatment and different spray schedules for the management of this disease.

\section{MATERIALS AND METHODS}

A field trial was conducted during kharif seasons of 2002 to 2004 (three years) at main farm of Oilseeds Research Station, Latur. Split plot design was used in the experiment. The experiment had two main treatments and seven sub-treatments replicated three times. Sunflower hybrid KBSH-1 was used for sowing. The net plot size of $3.0 \times 4.2 \mathrm{~m}$ was maintained for each treatment with $60 \mathrm{~cm}$ distance between rows and $30 \mathrm{~cm}$ between plants. All standard agronomic practices were applied. The treatment details are given below.

I Main treatments

$\mathrm{W}_{1}$ with sorghum border (6 rows)

$\mathrm{W}_{2}$ without sorghum border

II Sub treatments

$\mathrm{T}_{1}$ control

$\mathrm{T}_{2}$ seed treatment with imidacloprid (Gaucho 70 W.S.) at $5 \mathrm{~g} / \mathrm{kg}$

$\mathrm{T}_{3}$ spray of imidacloprid (Confidor 200 S.L.) at 0.05 percent, 15,30 and 45 DAS (days after sowing).

$\mathrm{T}_{4}$ spray of imidacloprid (Confidor 200 S.L.) at 0.05 percent, 15 and 45 DAS.

$\mathrm{T}_{5}$ seed treatment with imidacloprid (Gaucho 70 W.S.) at $5 \mathrm{~g} / \mathrm{kg}+$ spray of imidacloprid (Confidor 200 S.L.) at $0.05 \%, 15,30$ and 45 DAS $\left(T_{2}+T_{3}\right)$.

$\mathrm{T}_{6} \quad$ seed treatment with imidacloprid (Gaucho 70 W.S.) at $5 \mathrm{~g} / \mathrm{kg}+$ spray of imidacloprid (Confidor 200 S.L.), 15 and $45 \mathrm{DAS}\left(\mathrm{T}_{2}+\mathrm{T}_{4}\right)$.

$\mathrm{T}_{7}$ seed treatment with imidacloprid (Gaucho 70 W.S.) at $5 \mathrm{~g} / \mathrm{kg}+$ roguing of diseased plants. 
During the experiment, 6 rows of sorghum (variety PVK-801) were sown 15 day prior to sunflower sowing as a border crop around the main treatment $\left(\mathrm{W}_{1}\right)$. For sub-treatments, seed treatment and spray schedule were followed as described in treatment details. Percent necrosis incidence was recorded at two-week intervals till $60^{\text {th }}$ days of crop stand and mean percent of incidence was calculated. Yield data were recorded separately for each treatment after crop harvest. Treatment-wise mean percent necrosis incidence and yield data along with benefit/cost (BC ratio) are presented in Table 1. Percent necrosis incidence and year-wise yield data (2002-2004) are presented in Table 2.

Table 1: Effect of border treatment and sub-treatment on sunflower necrosis disease incidence and yield at ORS, Latur (Pooled 2002-2004)

\begin{tabular}{|c|c|c|c|c|c|c|c|c|}
\hline \multicolumn{2}{|c|}{ Sr. No. } & \multirow[t]{2}{*}{ Treatment details } & \multirow[t]{2}{*}{ 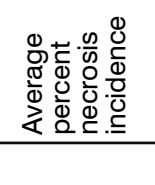 } & \multicolumn{2}{|c|}{ 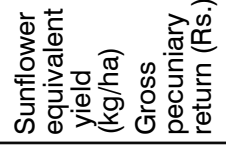 } & \multirow[t]{2}{*}{ 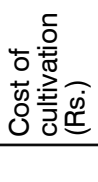 } & \multirow[t]{2}{*}{ 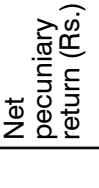 } & \multirow[t]{2}{*}{ 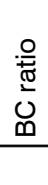 } \\
\hline \multirow[t]{5}{*}{$\mathrm{I}$} & \multicolumn{3}{|c|}{ Main treatment } & & & & & \\
\hline & $\mathrm{i}$ & With sorghum border $\left(\mathrm{W}_{1}\right)$ & $10.2(17.8)$ & 1222 & 19962 & 5869 & 14093 & 3.4 \\
\hline & ii & Without sorghum border $\left(\mathrm{W}_{2}\right)$ & $16.9(23.8)$ & 1030 & 16819 & 6000 & 10819 & 2.8 \\
\hline & & S.E. \pm & 0.5 & 5 & & & & \\
\hline & & C.D. (5\%) & 1.5 & 14 & & & & \\
\hline \multirow[t]{10}{*}{ II } & Sub & -treatment & & & & & & \\
\hline & $\mathrm{T}_{1}$ & Control & $22.3(28.0)$ & 849 & 13864 & 6000 & 7864 & 2.3 \\
\hline & $\mathrm{T}_{2}$ & $\begin{array}{l}\text { Seed treatment with } \\
\text { imdacloprid, } 5 \mathrm{~g} / \mathrm{kg}\end{array}$ & $18.6(25.3)$ & 930 & 15187 & 7550 & 8437 & 2.3 \\
\hline & $\mathrm{T}_{3}$ & $\begin{array}{l}\text { Spray of Confidor } 200 \text { S.L., } \\
0.05 \%, 15,30 \text { and } 45 \text { DAS. }\end{array}$ & $8.6(16.8)$ & 1238 & 20216 & 6650 & 13566 & 3.0 \\
\hline & $\mathrm{T}_{4}$ & $\begin{array}{l}\text { Spray of Confidor } 200 \text { S.L., } \\
0.05 \%, 15 \text { and } 45 \text { DAS. }\end{array}$ & $14.1(21.9)$ & 1081 & 17653 & 6370 & 11283 & 2.7 \\
\hline & $\mathrm{T}_{5}$ & $T_{2}+T_{3}$ & $4.9(12.1)$ & 1404 & 22927 & 7200 & 15727 & 3.1 \\
\hline & $\mathrm{T}_{6}$ & $\mathrm{~T}_{2}+\mathrm{T}_{4}$ & $9.5(17.4)$ & 1247 & 20363 & 6920 & 13443 & 2.9 \\
\hline & $\mathrm{T}_{7}$ & $\mathrm{~T}_{2}+$ roguing of diseased plants & $16.6(23.9)$ & 956 & 15611 & 7050 & 8561 & 2.2 \\
\hline & & S.E. \pm & 0.5 & 16 & & & & \\
\hline & & C.D. $5 \%$ & 1.3 & 47 & & & & \\
\hline \multicolumn{9}{|c|}{ III Interaction } \\
\hline & & S.E. \pm & 0.6 & 23 & & & & \\
\hline & & C.D. $5 \%$ & N.S. & N.S. & & & & \\
\hline
\end{tabular}

Figures in parenthesis are angular transformed values

1. Cost of sorghum production at Rs. 4000/ ha.

3. Price of sunflower produce at Rs. $1633 /$ qtls.

5. Cost of sorghum produce at Rs. 400/ qtls.
2. Cost of sunflower production at Rs. 6000/ ha.

4. Cost of imidacloprid (Gaucho) Rs. $100 / 5 \mathrm{~g}$.

6. Cost of imidacloprid (Confidor 200 S.L.) at Rs. 340/ 100 m.l. 


\section{RESULTS AND DISCUSSION}

The pooled data presented in Table 1 revealed that statically significant differences existed within main treatments. The main treatment, i.e., bordering of sorghum crop $\left(\mathrm{W}_{1}\right)$ recorded lowest mean necrosis incidence of 10.2 percent and maximum yield of $1222 \mathrm{~kg} / \mathrm{ha}$ along with highest $\mathrm{BC}$ ratio of 3.4 over non-border treatment $\left(\mathrm{W}_{2}\right)$. The former treatment $\left(\mathrm{W}_{1}\right)$ was also found to be significantly superior in respect to the latter treatment $\left(\mathrm{W}_{2}\right)$ in necrosis incidence as well as in yield. This indicated the effectiveness of border crop treatment in minimizing necrosis incidence and increasing crop yield. The sorghum border must have helped to minimize the frequency of vector flights from outside sources.

Table 2: Influence of border crop treatment and sub-treatment on sunflower necrosis incidence at Latur (2002-2004)

\begin{tabular}{|c|c|c|c|c|c|c|c|}
\hline \multirow{2}{*}{\multicolumn{2}{|c|}{ Treatment }} & \multicolumn{3}{|c|}{ Necrosis incidence (\%) } & \multicolumn{3}{|c|}{$\begin{array}{c}\text { Sunflower equivalent yield } \\
(\mathrm{kg} / \mathrm{ha})\end{array}$} \\
\hline & & \multirow[t]{2}{*}{2002} & \multirow[t]{2}{*}{2003} & \multirow[t]{2}{*}{2004} & \multirow[t]{2}{*}{2002} & \multirow[t]{2}{*}{2003} & \multirow[t]{2}{*}{2004} \\
\hline I & Main treatment & & & & & & \\
\hline i & With sorghum border $\left(\mathrm{W}_{1}\right)$ & $11.1(18.2)$ & 8.71 & $10.9(18.7)$ & 960 & 1390 & 1161 \\
\hline \multirow[t]{3}{*}{ ii } & Without sorghum border $\left(\mathrm{W}_{2}\right)$ & $19.3(25.7)$ & 14.29 & $17.4(24.1)$ & 801 & 1247 & 1034 \\
\hline & S.E. \pm & 0.6 & 0.1 & 0.7 & 26 & 38 & 29 \\
\hline & C.D. at $5 \%$ & 1.9 & 0.3 & 2.1 & 77 & 111 & 84 \\
\hline \multicolumn{8}{|c|}{ II Sub-treatment } \\
\hline $\mathrm{T}_{1}$ & Control & $25.1(29.9)$ & $19.14(25.71)$ & 22.9 & 650 & 1044 & 852 \\
\hline $\mathrm{T}_{2}$ & $\begin{array}{l}\text { Seed treatment with } \\
\text { imidacloprid, } 5 \mathrm{~g} / \mathrm{kg}\end{array}$ & $21.1(27.2)$ & $15.94(23.26)$ & 18.8 & 698 & 1170 & 921 \\
\hline $\mathrm{T}_{3}$ & $\begin{array}{l}\text { Spray of Confidor } 200 \text { S.L., } \\
0.05 \%, 15,30 \text { and } 45 \text { DAS. }\end{array}$ & $9.8(17.9)$ & $7.29(15.14)$ & $9.0(17.3)$ & 992 & 1454 & 1239 \\
\hline $\mathrm{T}_{4}$ & $\begin{array}{l}\text { Spray of Confidor } 200 \text { S.L., } \\
0.05 \%, 15 \text { and } 45 \text { DAS. }\end{array}$ & $14.5(22.1)$ & $12.54(20.55)$ & 15.5 & 825 & 1307 & 1107 \\
\hline $\mathrm{T}_{5}$ & $\mathrm{~T}_{2}+\mathrm{T}_{3}$ & $6.3(13.6)$ & $3.89(10.66)$ & $4.6(12.0)$ & 1182 & 1652 & 1371 \\
\hline $\mathrm{T}_{6}$ & $\mathrm{~T}_{2}+\mathrm{T}_{4}$ & $9.6(17.3)$ & $7.51(15.34)$ & $11.4(20.0)$ & 1063 & 1470 & 1204 \\
\hline \multirow[t]{3}{*}{$\mathrm{T}_{7}$} & $\mathrm{~T}_{2}+$ roguing of diseased plants & s $19.0(25.7)$ & $14.22(22.07)$ & $16.8(24.0)$ & 753 & 1130 & 983 \\
\hline & S.E. \pm & 1.1 & 1.2 & 1.1 & 53 & 87 & 52 \\
\hline & C.D. at $5 \%$ & 3.2 & 3.6 & 3.1 & 155 & 256 & 152 \\
\hline \multicolumn{8}{|c|}{ III Interaction } \\
\hline & S.E. \pm & 1.5 & 1.7 & & 75 & 123 & 74 \\
\hline & C.D. at $5 \%$ & N.S. & N.S. & N.S. & N.S. & N.S. & N.S. \\
\hline & C.V. (\%) & 12.6 & 16.2 & 12.1 & 14.9 & 16.3 & 11.6 \\
\hline & General Mean & 12.9 & 18.9 & 21.4 & 882 & 1318 & 1095 \\
\hline
\end{tabular}

Figures in parenthesis are angular transformed values.

Statistically significant differences were observed within sub-treatments. Amongst the sub-treatment, treatment $\mathrm{T}_{5}$ (seed treatment with imidacloprid + three sprays with Confidor) recorded lowest mean necrosis incidence of 4.9 percent, maximum yield of $1404 \mathrm{~kg} / \mathrm{ha}$ and highest $\mathrm{BC}$ ratio of 3.1 . This treatment was 


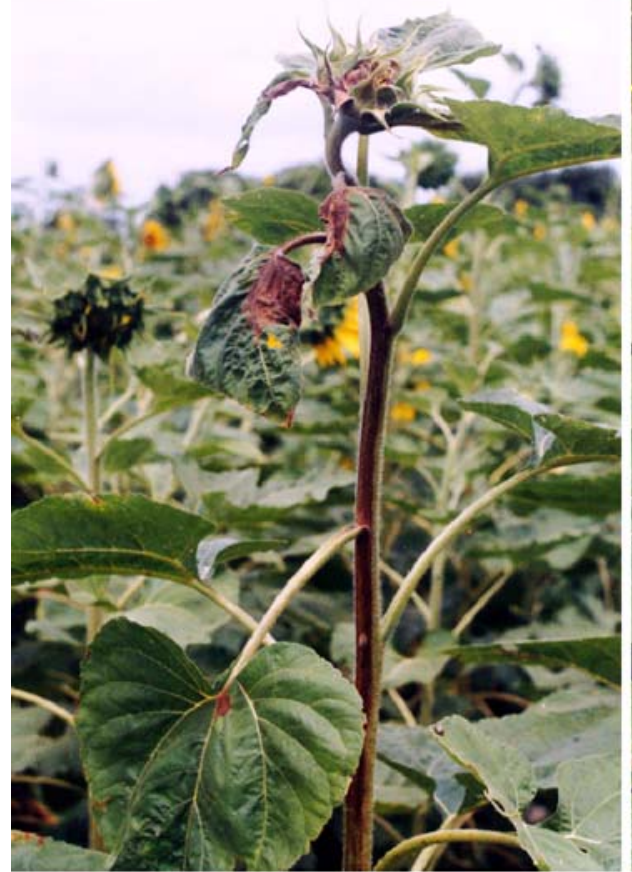

Figure 1: Stem blackening

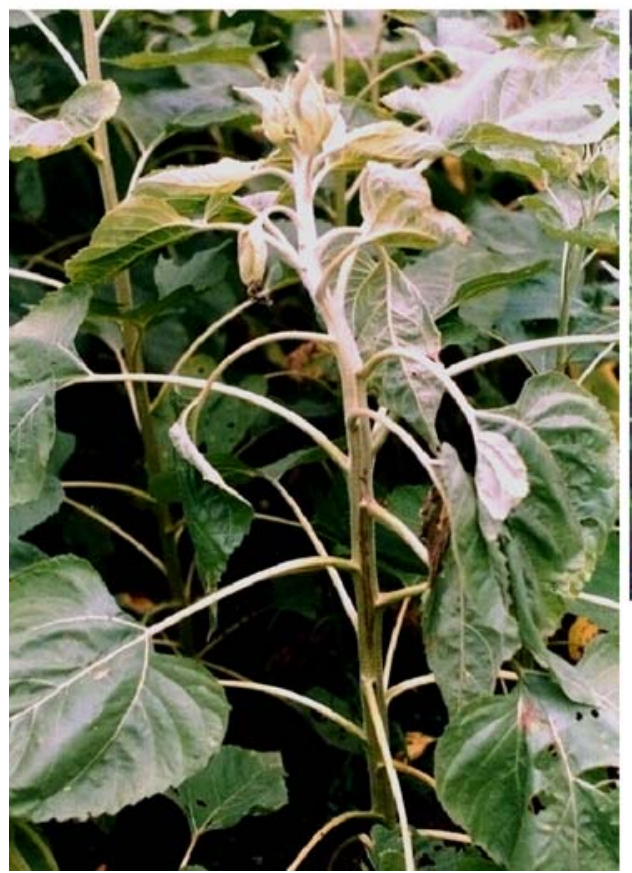

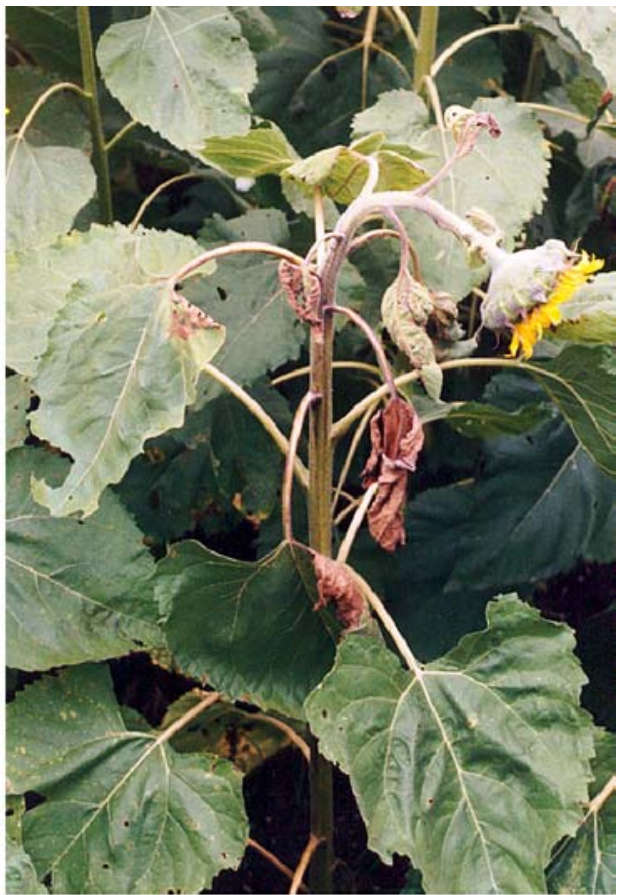

Figure 2: Necrosis of apical plant part

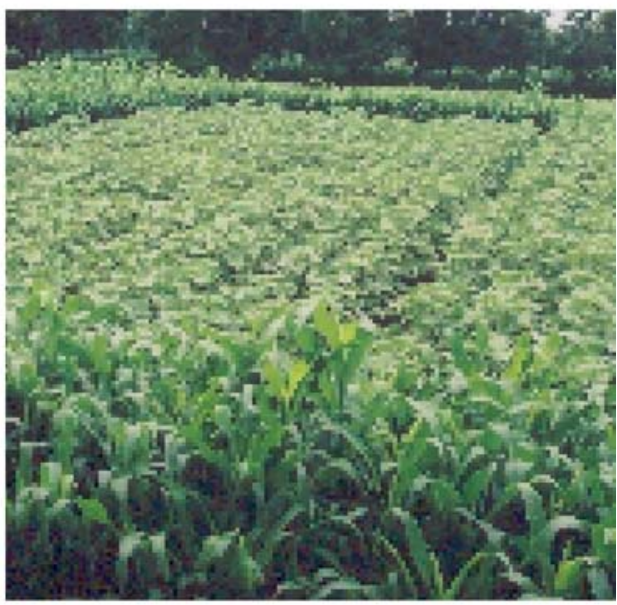

Figure 4: Low cost management of sunflower necrosis disease by growing sorghum around sunflower field

Figure 3: Typical stem bending 
also significantly superior over the other treatments. The interaction effects were non-significant. Thus it can be concluded from the above results that for the management of sunflower necrosis disease, the sunflower crop should be bordered with 6 rows of sorghum (border crop should be sown 15 day prior to main crop) and the sunflower seeds should be treated with imidacloprid (Gaucho 70 W.S., $5 \mathrm{~g} / \mathrm{kg}$ ) along with three sprays with imidacloprid (Confidor 200 S.L., 0.05 percent) applied 15, 30 and 45 days after sowing.

\section{ACKNOWLEDGEMENT}

The author is grateful to Dr. S.S. Kadam, Hon. Vice-Chancellor, MAU, Parbhani (M.S.), and Dr. D.M. Hedge, Project Director, DOR Hyderabad, India, for their encouragement and support.

\section{REFERENCES}

Bhat, A.I., Jain, R.K., Kumar, A., Ramiah, M. and Varma, A., 2002. Serological and coat protein sequence studies suggest that necrosis disease on sunflower in India is caused by a strain of tobacco streak ilar virus. Arch. Virol. 147: 651-658.

Chander, Rao, S., Raoof, M.A. and Singh, H., 2000. Sunflower necrosis disease; a preliminary study on transmission. In: Proceedings of Extended Summaries 2000. National Seminar on Oilseeds and Oilseeds Research Development Needs in the $3^{\text {rd }}$ Millennium, Indian Soc. of Oilseeds Res. (DOR), Hyderabad, w.e.f. February 2-4, 2000. pp: 285-286.

Harvir Singh, 2005. Thrips incidence and necrosis disease in sunflower (Helianthus annuus L.) J. Oilseeds Res. 22(1): 90-92.

Prasada Rao, R.D.V.J., Reddy, A.S., Chander Rao, S., Varaprasad, K.S., Thirumala Devi, K., Nagaraju Muniyappa, V. and Reddy, D.V.R., 2000. Tobacco streak ilar virus as a casual agent of sunflower necrosis disease in India. J. Oilseeds Res. 17(2): 400-401.

Shirshikar, S.P., 1997. Survey of sunflower downy mildew disease in Marathwada region. J. Maharashtra Agric. Univ. 22(1):135-136.

Shirshikar, S.P., 2003. Influence of different sowing dates on the incidence of sunflower necrosis disease. Helia 26(39): 109-116.

Shirshikar, S.P., 2005(a). Present status of sunflower downy mildew disease in India. Helia 28(43): 153-158.

Shirshikar, S.P., 2005(b). Control of downy mildew in sunflower with a new metalaxyl formulation (Apron XL-35 E.S.). Helia 26(39): 109-116.

Singh, S.J., Nagaraju, Krishna Reedy, M., Muniyappa, V. and Virupakashappa, K. 1997. Sunflower necrosis-a new virus disease from India. Annual Meeting of the Indian Phyto Pathological Society (IPS) West-zone meet on economically important diseases of crop plants, Bangalore, December 18-20, 1997. pp. 24.

\section{MANEJO INTEGRADO DE LA NECROSIS DEL GIRASOL}

\section{RESUMEN}

La necrosis del girasol (SND) es una nueva amenaza para el cultivo de girasol en India. Esta enfermedad se informó por primera vez en 1997 en Karnataka, uno de los estados que concentra mayor superficie de girasol en India. Más adelante se informó su ocurrencia en la mayoría de los estados de India, convirtiéndola en una amenaza para el cultivo de girasol. 
Actualmente no hay disponibles fuentes confiables de resistencia. Dado que la enfermedad es de naturaleza viral es muy difícil de combatir a través de una aproximación simple. Tres años de experimentos (2002-2004) conducidos en la Oilseeds Research Station, Latur (M.S.) India, revelaron que si el cultivo de girasol está rodeado de sorgo y las semillas de girasol se tratan con imidacloprid (Gaucho 70 W.S.) at $5 \mathrm{~g} / \mathrm{kg}$ además de la pulverización del cultivo con imidacloprid (Confidor 200 S.L.) at 0.05\%-tres aplicaciones a los 15, 30 y 45 días después de la siembra) se puede minimizar la incidencia de la necrosis del girasol.

\section{CONTRÔLE INTÉGRAL DE LA NÉCROSE DU TOURNESOL RÉSUMÉ}

La maladie nécrotique du tournesol est un nouveau dommage pour la culture du tournesol en Inde. La maladie a été répertoriée en 1997 dans le Karnataka, un état majeur de l'Inde pour la culture du tournesol. Plus tard, son apparition a été reportée dans la plupart des zones de culture du tournesol en Inde ne causant des dégâts sérieux.

A l'heure actuelle, aucune source de résistance n'est disponible. Du fait que la maladie est de nature virale, il est très difficile de la combattre par une approche simple. A la Station de Recherche sur les Oléagineux de Latur (M.S., Inde], une expérimentation au champ de trois ans (2002-2004) a révélé que si la culture du tournesol est bordée par du sorgho, si les semences de tournesol sont traitées avec l'imidacloprid (Gaucho 70 W.S.) à $5 \mathrm{~g} / \mathrm{kg}$ et que la culture est traitée en spray avec l'imidacloprid (Confidor 200 S.L.) à la dose de 0.05 pour cent (trois sprays à 15, 30 et 45 jours après le semis), l'incidence de la maladie nécrotique du tournesol peut être minimisée. 
